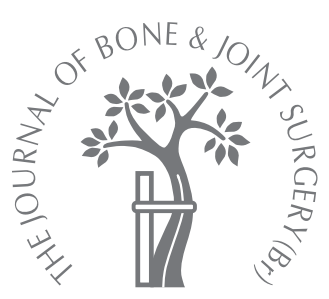

N. Jain,

K. M. Willett

From The Kadoorie

Centre, John

Radcliffe Hospital,

Oxford, England
N. Jain, MS(Orth), Senior House Officer

Accident and Emergency Medicine

Cumberland Infirmary, Carlisle CA2 7HY, UK.

K. M. Willett, FRCS, Professor of Orthopaedic

Trauma Surgery

Kadoorie Centre

John Radcliffe Hospital,

Oxford OX3 9DU,

Oxfordshire, UK.

Correspondence should be sent to Mr K. M. Willett;

e-mail:

keith.willett@ndos.ox.ac.uk

C2006 British Editorial Society of Bone and Joint Surgery

doi:10.1302/0301-620X.88B1. $17158 \$ 2.00$

$J$ Bone Joint Surg $[\mathrm{Br}]$ 2006:88-B:111-15.

Received 2 September 2005; Accepted 20 September 2005

\title{
Maintaining standards in surgical training
}

\author{
HOW EFFECTIVE IS THE ACCREDITATION BY THE SPECIALIST \\ ADVISORY COMMITTEE IN THE UNITED KINGDOM?
}

In order to assess the efficacy of inspection and accreditation by the Specialist Advisory Committee for higher surgical training in orthopaedic surgery and trauma, seven training regions with 109 hospitals and 433 Specialist Registrars were studied over a period of two years.

There were initial deficiencies in a mean of $14.8 \%$ of required standards $(10.3 \%$ to $19.2 \%)$. This improved following completion of the inspection, with a mean residual deficiency in $8.9 \%(6.5 \%$ to $12.7 \%$.) Overall, $84 \%$ of standards were checked, $68 \%$ of the units improved and training was withdrawn in $4 \%$.

Most units $(\mathbf{9 7} \%)$ were deficient on initial assessment. Moderately good rectification was achieved but the process of follow-up and collection of data require improvement. There is an imbalance between the setting of standards and their implementation. Any major revision of the process of accreditation by the new Post-graduate Medical Education and Training Board should recognise the importance of assessment of training by direct inspection on site, of the relationship between service and training, and the advantage of defining mandatory and developmental standards.

Prior to 30 September 2005 in the United Kingdom a Specialist Advisory Committee (SAC) in each surgical specialty reported to the Specialist Training Authority (STA) of the Medical Royal Colleges through the Joint Committee of Higher Surgical Training (JCHST) and set the standards for Higher Surgical Training (HST). ${ }^{1,2}$ The SACs also monitored the effective implementation of these standards through visits of inspection. Although this system had worked, there were concerns that, in the absence of written guidelines, the SACs applied variable and inconsistent standards. Furthermore, when training units fell short of the required standards, there were variations in how they were rectified and what sanctions were applied.

In February 2001 the standards for HST in trauma and orthopaedic surgery ${ }^{3}$ were published against which individual units and training programmes would be assessed. These were revised in June 2002, April 2003 and January 2004. The standards selected had been refined over many years of experience of visits and were specific to the specialty.

Using these standards, requirements or recommendations were produced after visits of inspection. A requirement was defined as a change in service, organisation, practice or resource which was considered essential to fulfil the core training needs in a programme and its component units in order to maintain accreditation and keep specialist registrars. These are considered as 'musts' in the standards for HST. A recommendation was defined as a change in service, organisation, practice or resource which would improve or resolve weaknesses in a programme which was otherwise considered adequate. These were characterised under 'should'.

The standards are applicable either to the whole training programme or to individual training units. Those which apply to units are classified into four groups: a) infrastructure and facilities, b) staffing and team structure, c) in-service training and d) education, with requirements and recommendations for each group. There was a total of 45 requirements and 28 recommendations.

Standards were also introduced for the conduct and process of the visits of inspection and reports. ${ }^{4}$ There was to be a quinquennial visit of inspection with follow-up visits and progress reports. Units submitted a Hospital Application Form to the JCHST at least one month in advance of the visit, which was conducted by at least two members of the SAC. They were briefed in advance by the Hospital 
Application Form, reports of previous inspections and assessment forms completed by the trainees from the RITA (Regional In-Training Assessment) process. The visit lasted for at least half a day and included a tour of the facilities and meetings with the programme director, the SAC liaison member, consultant trainers, hospital senior management, and interviews with each trainee.

Once ratified by the SAC, the visitors' report was distributed by the JCHST to the programme director and lead consultant. It conformed to a standard template and listed requirements and recommendations to rectify unmet standards, when the next review would take place and what sanctions would be applied if the proposed changes had not been achieved.

We have evaluated the SAC inspection system in order to measure its efficacy in monitoring and implementing standards. We have assessed the performance of this accreditation body and hope that our findings may contribute to the major revision of the process of inspection with the inception of the Post-graduate Medical Education and Training Board (PMETB). ${ }^{5}$

\section{Training Programmes and Regions Assessed}

Training units of regional programmes were visited after the standards were agreed in February 2001. The regions studied were North Thames East (12 units), North Thames West (17), South Thames East (17), and the West Midlands (19) in England, Aberdeen, Dundee and Edinburgh (10) in Scotland, and the programmes covering Wales (6) and the Republic of Ireland (17). Although the SAC inspects training in the Republic of Ireland, the STA has no legal authority in that country.

We studied reports of the quinquennial visits and progress reports submitted by programme directors, SAC liaison members and training units regarding compliance with the requirements, together with reports of revisits to the units. The training experiences of 433 higher surgical trainees were assessed. Each report was scored against the published standards, but analysis of the administration of the programmes was not included. When this study began there was some overlap of standards and so a list of 31 requirements and 19 recommendations was selected.

Where a standard was not checked, it was assumed to have been unmet.

Outcome measures. The primary outcome measure was the reduction in the number of unmet requirements when the process of inspection/rectification had been completed. Secondary outcomes were the proportion of applicable standards checked and the frequency of deficiencies in each component of training. Collection and analysis of the data was undertaken by the author without prior knowledge of previous performances and information was only obtained from reports that had been submitted to the JCHST.

Statistical analysis was carried out using the Student's $t$ test and the Mann-Whitney-Wilcoxon tests. The deficien-
Table I. Mean and range of recommendations found inadequate by region

\begin{tabular}{ll}
\hline Region & Mean (range) \\
\hline North Thames East & $3(0$ to 7$)$ \\
North Thames West & $3(0$ to 7$)$ \\
South Thames East & $3(0$ to 7$)$ \\
West Midlands & $3(0$ to 6$)$ \\
Scotland & $2(0$ to 8$)$ \\
Wales & $3(1$ to 6$)$ \\
Republic of Ireland & $4(1$ to 7$)$ \\
\hline
\end{tabular}

cies and effectiveness of rectification were compared across the training regions.

\section{Results}

We analysed 98 quinquennial reports, 31 reports of revisits and 64 assessments covering 109 training units, of which 79 had both trauma and elective elements, 13 had only trauma and 17 only elective orthopaedics.

\section{Primary outcome measure}

The reduction in the number of unmet requirements in all the training units over a period of two years. In all but three units, there was at least one standard of training unmet sufficient to warrant a requirement to change (mean 4.6; SD 2.4; range 0 to 12). At the time of initial inspection there were deficiencies in a mean of $14.8 \%$ of standards by region $(10.3 \%$ to $19.2 \%)$. This represented a failure to apply 450 of the total of 3038 absolute 'must' requirements assessed. This improved after the inspection process, with a mean residual deficiency in $8.9 \%$ of standards by region $(6.5 \%$ to $12.7 \%)$. The total number of problems which had not been rectified was 270 . Eleven units had no outstanding requirements; in a further 61 units deficiencies were rectified, leaving a mean of 2.8 (SD 2.1, 0 to 7) unmet needs in the remaining units. Application of the Student's $t$-test and the Mann-Whitney-Wilcoxon test to the initial visit and outcome data gave $p$ values of $<0.05$. An analysis of the 24 recommendations revealed similar results (Table I).

The outcome of the rectification of requirements in individual units was divided into five categories: no follow-up necessary, all unresolved, some partially resolved, adequately resolved and training withdrawn.

In some units the number of trainees was reduced to rectify specific deficiencies in training but the units remained accredited. These were included in the adequately-resolved category. The 'no follow-up necessary' group included those units which did not have any identified deficiencies or those which were solved during the visit.

Recognition of training was withdrawn from $4 \%$ of units. The requirements were resolved in $51 \%$ of the units and partially resolved in $17 \%$. In $27 \%$ the requirements were not recorded as resolved. In these units either followup was not documented as complete $(14 \%)$, or no followup was asked for despite requirements at the end of the visit $(13 \%)$. An analysis by country was performed (Fig. 1). 


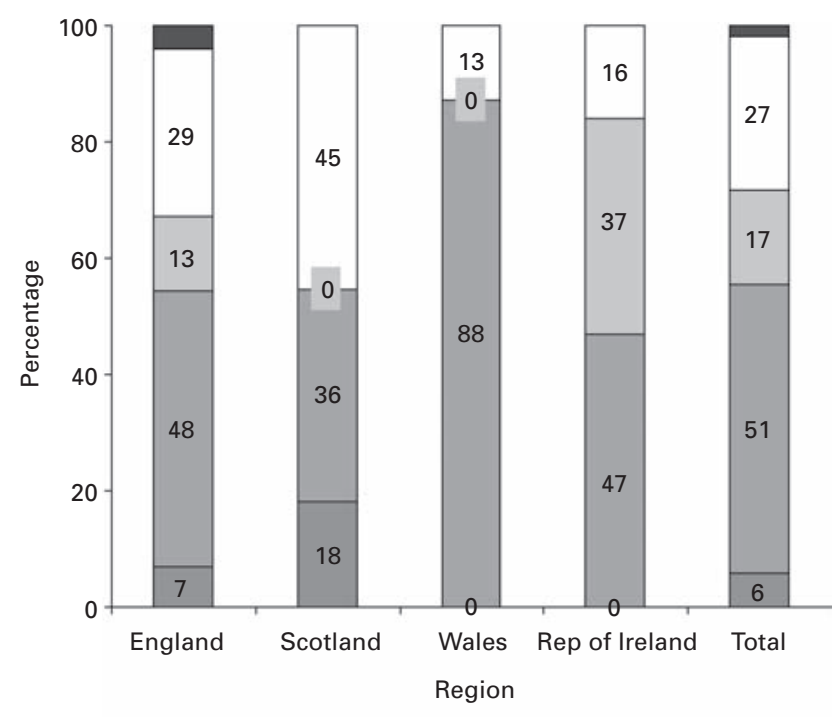

ㅁ Problems identified but not recorded as resolved

- Problems identified and partially resolved

- Problems identified and adequately resolved

- No follow-up necessary

- Training withdrawn

Fig. 1

Percentage rectification of requirements by country.

\section{Secondary outcome measures}

The proportion of applicable standards recorded as checked. The percentage of standards checked in the units showed a mean of $84 \%$ ( $71 \%$ to $100 \%)$. The regional variation was similar (Table II).

Analysis of standards by region which have not been recorded shows that $16 \%(11 \%$ to $19 \%)$ would have generated requirements and $14 \%(11 \%$ to $15 \%)$ recommenda-
Table II. Standards recorded as checked by region

\begin{tabular}{ll}
\hline Region & Mean (range) \\
\hline North Thames East & 85 (78 to 92) \\
North Thames West & 83 (76 to 94) \\
South Thames East & 81 (74 to 94) \\
West Midlands & 86 (74 to 93) \\
Scotland & 89 (74 to 100) \\
Wales & 86 (84 to 93) \\
Republic of Ireland & 83 (71 to 98)
\end{tabular}

tions. Some requirements, such as teaching application of casts and the availability of study leave with funding, were frequently missing from the reports. These are not listed on the Hospital Application Form.

The frequency of deficiencies in each component of training. The deficiency in standards before rectification was further analysed to identify the problem areas of training within and between training regions (Fig. 2). The greatest number of problems was in the Republic of Ireland, in the main related to the timetables of the trainees with an imbalance between clinics and theatre time and poor provision for release to the post graduate programme. This was identified by visitors to be a result of the slower implementation of working time directives. At the time of the study there remained a dominance of service provision over training in the Republic of Ireland. Key indicator standards of the commitment to training varied across units and nationally (Fig. 3).

The units providing training in orthopaedic trauma have a mean of 1.4 trauma operating sessions per trainee per week. This is above the requirement of at least one trauma list per week and is maximum for the Republic of Ireland at 2.4 and least for England at 1.2. Similarly sessions in the fracture clinic per week per trainee are at a mean of $1.3(1.2$ to 1.8 ).

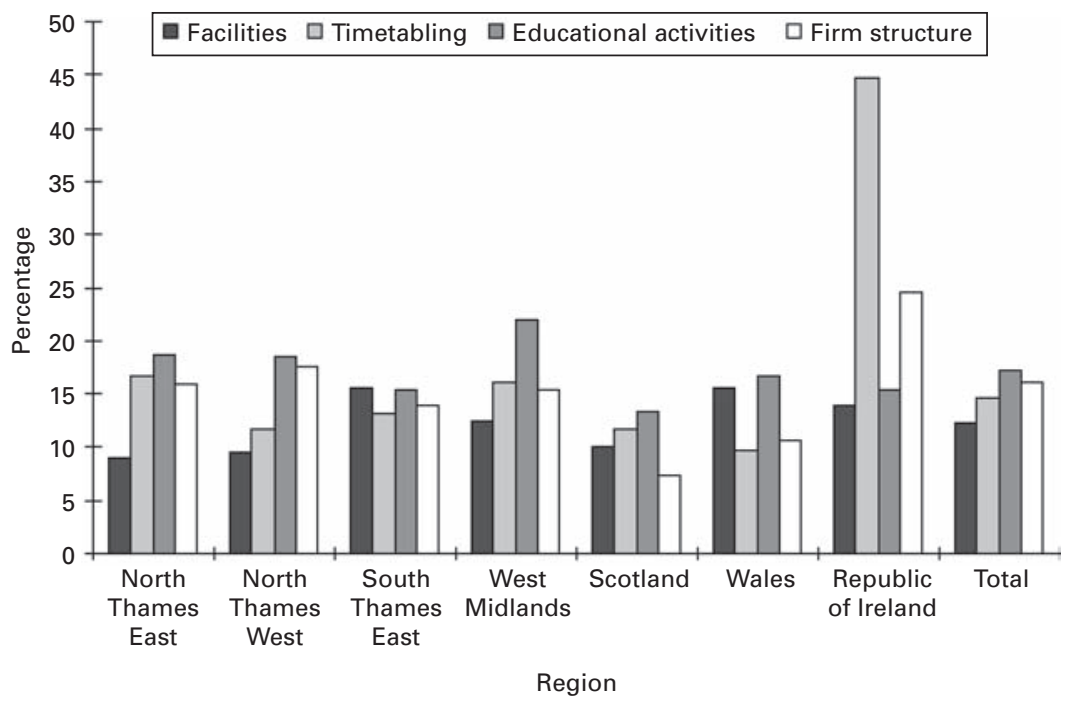

Fig. 2

Problem areas identified in training by region. 


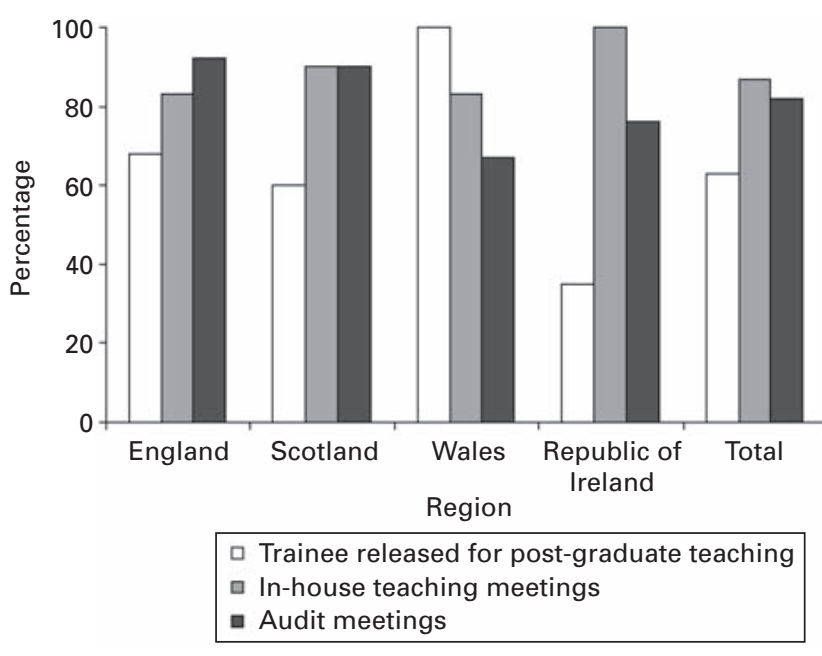

Fig. 3

Teaching activity in training units.

The provision of weekend trauma lists $(22 \%$ of units, $6 \%$ to $27 \%)$ and daily trauma meetings $(62 \%, 18 \%$ to $82 \%$ ) are most variable. Trauma surgery on weekend days is often managed on emergency lists with less likelihood of supervision and has to compete with other surgical emergencies.

The training time in elective orthopaedics is better than that in trauma. Trainees have a mean 2.8 elective lists (2.6 to 3.5 ) and 2.1 elective clinics (1.9 to 2.5 ) per week per trainee.

\section{Limitations of the study}

The data were only collected from completed reports submitted to the JCHST and the authors recognise that SAC liaison members may have held additional knowledge of rectification. The Hospital Application Form used during the period of the study, and the standardised training unit report, were developed prior to publication of the training standards and did not record all of them. The documentation of compliance for some standards was dependent on the diligence of the site visitors. Unrecorded or unchecked standards were assumed not to have been met.

\section{Other national accreditation processes}

The inspection and the standards for accreditation of training posts work differently in other countries. In Australia and New Zealand, the system of accreditation ${ }^{6}$ is carried out by the Specialist Education Accreditation Committee of the Australian Medical Council. The process starts with the training organisation submitting an accreditation submission, which is a self-study guided by a detailed list of questions or guidelines. The Board of Advanced Surgical Training then forms a visiting team of two board members and one jurisdictional representative which assesses the training posts and submits a report. If accepted, the decision could be for a six-year accreditation for higher specialist training. An annual report is then required. A comprehensive report half way through the period of accreditation should provide assurance and, where possible, evidence that standards of education and training and appropriate resources have been maintained. The goals and objectives are derived from the work done by the Royal College of Physicians and Surgeons of Canada (RCPSC) CanMEDS 2000 project. Training programmes in Canada are University-based; trainees may spend up to half their training in peripheral units provided the curriculum and syllabus are covered. The process ${ }^{7}$ in Canada is more comprehensive than that in the United Kingdom and has a sixyear cycle with an external review by the RCPSC in year one, and a University review in year four. A survey team, drawn from another specialty, checks the administration of the programme, general education and specialty-specific standards. The team undertakes the Royal College site visit and recommends to the College the category of accreditation.

The American College of Surgeons has a similar measurement of publicly-available standards of accreditation. ${ }^{8}$ A programme can expect to be visited every three to four years. Significantly the site-visitor is a fact-finder only and verifies the information submitted on the Programme Information Form. The decision for accreditation lies with the Accreditation Council for Graduate Medical Education. The American system, in common with the others described above, has extensive and detailed education and general organisational requirements but is not as specific for infrastructure, clinical team-working or service experience as those in the United Kingdom. A review of current documentation from these accreditation bodies suggests that the processes are all evolving or under review.

\section{Discussion}

Throughout the developed world, accreditation for training has developed to some level. However, the assessments remain unchecked and their performance unmeasured. There is a constant drive to revise established systems. The external demands for such changes include the level of competence expected of trainees, quality assurance in education and constraints from working time directives. There are governmental influences on the size of the specialist workforce required and the end-of-training experience appropriate to the system of health care in the individual country. The merging of national training standards, as witnessed in Europe, creates further regulation and may generate the need for modification of training to achieve compliance.

A review of the methods of accreditation in different countries shows strong similarity with a recognition of the need to measure the educational structure and organisation of the programme, its system of internal review and, for each post, the key requirements for clinical and academic opportunities. A site inspection in order to validate submitted data is a uniform component. The level of specialtyspecific inquiry varies, as does the involvement of lay members or representatives of other specialties. The concept of 
separating requirements to achieve adequate training from recommendations to achieve best practice and the process of rectification are less well described.

Our study has evaluated the efficacy of such an accreditation system. Derivation of standards has been common to all systems in the last decade with a tightening of the criteria, particularly in recent years. An important function of the inspection, in addition to checking standards of training, is to assess the service pressures which may frequently detract from the opportunities for training. However, the primary purpose of the accreditation process and of our study is to identify the ability to rectify deficiencies in training. Our findings support the definition of requirements ('musts') and recommendations ('shoulds') in setting standards successfully and achieving clarity between those elements that are mandatory for current training and would provoke sanctions or withdrawal of approval if deficient, and those which would develop best practice.

Overall the mean deficiency in meeting minimum training standards was $14.8 \%$, with the worst unit being $38.7 \%$, suggesting a strong need for inspection and monitoring. The United Kingdom's system of control by the SAC addressed those deficiencies, completely or partially, in $73 \%$ of units. The standards set are very specific for a surgical specialty and few units were achieving them on the initial inspection. If these are minimum standards, then partial achievement is unacceptable unless, as was apparent from the reports, good quality training was still occurring in some units. Failure on multiple standards or key standards indicated a poor commitment to training.

The SAC inspection appears reasonably complete in that $84 \%$ of requirements were checked. If the standards which were not in the Hospital Application Form-generated report are excluded, this increases to $97 \%$. A redesign of this form specific to the measured standard is strongly recommended. The high compliance with certain standards suggests that they may be poor indicators or could be assessed without a site visit.

However, there remained 26 units in which the required change was not achieved within the period of study. In these the process of rectification is demonstrably less robust. This may be a feature of organisation in prompting reviews and documentation, delegation to individuals, the loss of priority over future inspections of other programmes or the rotation of SAC members. The quinquennial visits, in contrast, are highly organised full committee activities. Rectification needs improvement and its weakness is probably due to the evolution of the process of quality assurance in which the setting and measurement of standards have primacy and the review and sanction procedures are secondary. This may be improved by either strengthening the procedures and priority afforded to follow-up or devolving such responsibility to the accountable Deanery.

In the United Kingdom, supplementary data on training are available from the RITA programme but are poorly integrated into the process of accreditation. In the United States a rate of return of at least $70 \%$ of questionnaires is required from residents before a programme accreditation can be considered. Information from trainees at RITA assessments should more directly feed the process of inspection.

In comparison with other countries, the United Kingdom's system, while it retains programme administration, academic, trainee assessment and research requirements, favours clinical activity and standards of supervision specific to the specialties. In order to ensure effective quality assurance, the standards set for training programmes for administration, education resource, curriculum delivery and trainee performance must be specific to measure the training required in clinical practice. This would appear to be crucial in the United Kingdom given the current concerns in the transition to Modernising Medical Careers. ${ }^{9}$ Wholesale changes in the accreditation process, without retention of the proven core of the SAC system, would be hazardous. To measure clinical exposure and training, the site-visit and interrogation of the service unit should remain. Detailed inquiry of the inter-relationship between trainers and trainees within a clinical firm is required to clarify the quality of, and exposure to, clinical training. This was apparent in the data analysed for our study. The need for this level of interrogation is arguably stronger in the craft specialties. The authors would suggest due caution in any revision of the accreditation process without proper validation.

\section{Supplementary Material}

远 A list of the training standards used for individual training units is available with the electronic version of the article on our website at www.jbjs.org.uk

No benefits in any form have been received or will be received from a commercial party related directly or indirectly to the subject of this article.

\section{References}

1. Specialist Training Authority. General information. Information Sheet 1. http:// www.jchst.org (accessed 01/06/05).

2. Specialist Training Authority. Policy statement; sanctions and withdrawal of training recognition from training units. http://www.jchst.org (accessed 01/06/05)

3. SAC in Trauma and Orthopaedics. Standards of higher surgical training February 2001. http://www.jchst.org (accessed 01/04/05).

4. SAC in Trauma and Orthopaedics. Standards for visits, reports and sanctions February 2001. http://www.jchst.org (accessed 01/04/05).

5. Department of Health. Postgraduate medical education and training, The Medical Education Standards Board: a paper for consultation, Nov 2001. http:// www.dh.gov.uk/assetRoot/04/02/11/91/04021191.pdf (accessed 01/06/05).

6. Accreditation of Specialist Medical Education and Training and Professional Development Programs. Standards and procedures, Australian Medical Council July 2003. http://www.amc.org.au/forms/guidelines.pdf (accessed 01/06/05).

7. Royal College of Physicians and Surgeons of Canada Residency Programme Accreditation 1996. www.rcpsc.medical.org/residency/accreditation/index.php (accessed 01/06/05)

8. (American) Accreditation Council for Graduate Medical Education. www.acgme/org (accessed 01/06/05).

9. The next steps - The future shape of foundation, specialist and general practice training programmes. NHS Modernising Medical Careers 15 April 2004 www.mmc.nhs.uk (accessed 01/06/05). 